\title{
Clinical Significance of Smudge Cells in Peripheral Blood Smears in Hematological Malignancies and Other Diseases
}

\author{
Chih-Chun Chang ${ }^{1 \star}$, Jen-Tang Sun ${ }^{2 \&}$, Tse-Hsuan Liou ${ }^{1 \&}$, Chin-Fu Kuo ${ }^{1}$, Chia- \\ Hao Bei ${ }^{1}$, Sheng-Jun Lin ${ }^{1}$, Wei-Ting Tsai ${ }^{1}$, N-Chi Tan ${ }^{1}$, Ching-Biau Liou ${ }^{1}$, Ming- \\ Jang $\mathrm{Su}^{1}$, Tzung-Hai Yen ${ }^{3}$, Fang-Yeh $\mathrm{Chu}^{1,4,5 *}$
}

\begin{abstract}
Background: It is reported that the percentage of smudge cells in the blood smear could be a prognostic indicator in chronic lymphocytic leukemia. However, the clinical significance of smudge cells in other hematological malignancies, solid tumors or non-malignant diseases is less clear. Hence, this study was conducted to survey the clinical significance of smudge cells in hematological cancers and other disorders. Materials and Methods: From January to November, 2015, the clinical data of patients who received blood examination with differential counts for clinical purpose and were found to have smudge cells in the peripheral blood film in Far Eastern Memorial Hospital were selected. The percentage of smudge cells and patient outcomes were evaluated for further univariate and survival analyses. Results: A total of 102 patients with smudge cells in their blood smears were included. Smudge cells were frequently presented in out-of-hospital cardiac arrest $(\mathrm{OHCA} ; \mathbf{n = 3 0})$, infections $(n=23)$, hematological cancers $(n=23)$ and solid cancers $(n=10)$. There was no relationship between the percentage of smudge cells and the patient mortality in all diseases (OR: 1.08, 95\% CI: $0.47-2.48, P=1.000)$ as well as the OHCA group (OR: $1.91,95 \%$ CI: $0.38-9.60, P=0.694)$. It was observed that in patients with all cancers with the percentage of smudge cells less than $50 \%$ had a lower mortality rate in comparison with those who had the percentage of smudge cells of 50\% or more (OR: $22.29,95 \%$ CI: $2.38-208.80, P<0.001)$. Additionally, it was seemingly that patients with smudge cells of $50 \%$ or more had a lower survival rate than those with smudge cells less than 50\% in all cancers with follow-up at 2-month intervals, but without statistical significance $(P=0.064)$. Conclusions: Our survey indicated that in all cancers, those who had higher percentage of smudge cells were prone to have poor outcomes when compared with the subjects with lower percentage of smudge cells. This finding was quite different from the results of previous studies in which the race-ethnicity of most study populations was non-Asian; hence, further investigations are required. Besides, there was no apparent association of the percentage of smudge cells with patient outcomes in all diseases, including OHCA.
\end{abstract}

Keywords: Smudge cells - chronic lymphocytic leukemia - hematological malignancies - cardiac arrest

Asian Pac J Cancer Prev, 17 (4), 1847-1850

\section{Introduction}

Smudge cells, or the so-called Gumprecht shadows, are ruptured or damaged leukocytes during the slide preparation of peripheral blood. Smudge cells may also be presented as bare nuclei isolated from the broken cells varying from well-preserved nuclei without cytoplasm, to smudged nuclear materials with the arrangement of strands in a coarse network, and therefore named basket cells. It has been considered that the formation of smudge cells is attributed to one of the artifacts in vitro in preparing the peripheral blood smear, probably because of the fragility of the leukocytes (Gumprecht, 1986; Macdonald et al., 2003). In case of atypical lymphocytosis such as chronic lymphocytic leukemia or acute leukemic changes, it could be observed that numerous smudge cells presented in the blood film. Recently, it has been suggested that smudge cells are constituted of apoptotic leukocytes dying in circulation, representing the auto-suicide of cells in process (Paydas, 2010). Further investigation revealed that the percentage of smudge cells could be a prognostic indicator in patients with chronic lymphocytic leukemia (Nowakovski, 2009; Johansson, 2010). However, the clinical significance of smudge cells in other hematological

${ }^{1}$ Department of Clinical Pathology, ${ }^{2}$ Department of Emergency, Far Eastern Memorial Hospital, New Taipei, ${ }^{3}$ Division of Nephrology and Clinical Toxicology, Chang Gung Memorial Hospital, Lin-Kou Medical Center, ${ }^{5}$ Graduate School of Biotechnology and Bioengineering, Yuan Ze University, Taoyuan, ${ }^{4}$ School of Medical Laboratory Science and Biotechnology, Taipei Medical University, Taipei, Taiwan ${ }^{\star}$ Equal contributors *For correspondence: jacpha@mail.femh.org.tw 
malignancies, solid tumors or non-malignant diseases remains to be determined. Therefore, we conducted this study to survey the clinical significance of smudge cells in hematological cancers and other disorders.

\section{Materials and Methods}

From January to November, 2015, patients who had archived examination of complete blood count and differential count for clinical purpose revealing the presence of smudge cells in the peripheral blood film in Far Eastern Memorial Hospital were selected. The clinical data were requested, including patient age, gender, clinical diagnosis, absolute lymphocyte count, the percentage of smudge cells and the patient outcomes. The percentage of smudge cells was calculated as the ratio of smudged to intact cells plus smudged lymphocytes. The initial data would be adopted when a patient had multiple results concerning the percentage of smudge cells. Patients would be followed up in a 2-month episode individually for the monitoring of co-morbidity and mortality if any.

Statistical analysis was performed using SPSS (version 19.0; SPSS Inc., Chicago, USA) statistical software. All data were expressed as the median (interquartile range; IQR) or case number (percentage). The odds ratio (OR) with $95 \%$ confidence interval (CI) was calculated using the unconditional logistic regression model in assessment of the relationship of percentage of smudge cells to patient outcomes in hematological cancers and other diseases. The cut-off value of the percentage of smudge cells was determined using the integral number near the median of each disease in categorization. The survival analysis was executed using Kaplan-Meier method with log-rank test. Statistical significance was considered if a $\mathrm{P}$ value was less than 0.05 .

\section{Results}

During the study episode, a total of 102 patients who were found to have smudge cells in the peripheral blood smear at Far Eastern Memorial Hospital were enrolled. The demographic features of these subjects were listed in Table 1. Among these, 30 (29.4\%) were admitted due to out-of-hospital cardiac arrest (OHCA), $23(22.5 \%)$ were hematological malignancies and another $23(22.5 \%)$ had infections, $10(9.8 \%)$ had solid cancers and $16(15.7 \%)$ belonged to miscellaneous. The median of absolute lymphocyte count was $4.3(1.6-6.9) \times 10^{3} /$ $\mathrm{uL}$ in all diseases, $5.5(2.6-6.8) \times 10^{3} / \mathrm{uL}$ in OHCA, 2.5 $(0.9-10.1) \times 10^{3} / \mathrm{uL}$ in all cancers, $3.5(1.2-11.6) \times 10^{3} / \mathrm{uL}$ in hematological cancers, and $5.0(1.8-9.4) \times 10^{3} / \mathrm{uL}$ in infections, respectively. The absolute lymphocyte count was seemingly higher in OHCA and infections and lower in cancer groups, but with no statistical significance. Besides, the median percentage of smudge cells was 34.5 (22-55) \% in all diseases, 25.5 (19-34) \% in OHCA, 54 (29.5-67) \% in all cancers, 50 (28-71) \% in hematological malignancies, and 34 (22-46) \% in infections, respectively. Eventually, the overall 2-month mortality was $34.3 \%$ in patients who had smudge cells in the blood film.

Univariate analysis for the percentage of smudge cells and patient outcomes in those with smudge cells in the peripheral blood smear were shown in Table 2. There was no relationship between the percentage of smudge cells and the patient mortality in all diseases (OR: 1.08, 95\% CI: $0.47-2.48, \mathrm{P}=1.000$ ) as well as the $\mathrm{OHCA}$ groups (OR: $1.91,95 \%$ CI: $0.38-9.60, \mathrm{P}=0.694)$ at the cut-off level of $50 \%$ of smudge cells. It was also observed that patients in all cancers with the percentage of smudge cells less than $50 \%$ had a lower mortality rate in comparison with those who had the percentage of smudge cells of $50 \%$ or more (OR: $22.29,95 \%$ CI: $2.38-208.80, \mathrm{P}<0.001)$. The

Table 1. The Demographic Characteristics in Patients with Smudge Cells in the Peripheral Blood Film

\begin{tabular}{lc}
\hline Variables & \\
\hline Age (year) & $60(37-75)$ \\
Gender (Male/Female) & $61 / 41$ \\
Diseases & $30(29.4 \%)$ \\
OHCA & $23(22.5 \%)$ \\
Hematological malignancies & $23(22.5 \%)$ \\
Infections & $10(9.8 \%)$ \\
Solid cancers & $16(15.7 \%)$ \\
Miscellaneous & \\
Absolute lymphocyte count (x1000/uL) & $4.3(1.6-6.9)$ \\
All diseases & $5.5(2.6-6.8)$ \\
OHCA & $2.5(0.9-10.1)$ \\
All cancers & $3.5(1.2-11.6)$ \\
Hematological malignancies & $5.0(1.8-9.4)$ \\
Infections & $34.5(22-55)$ \\
The percentage of smudge cells (\%) & $25.5(19-34)$ \\
All diseases & $54(29.5-67)$ \\
OHCA & $50(28-71)$ \\
All cancers & $34(22-46)$ \\
Hematological malignancies & $34.3 \%$ \\
Infections & \\
Overall mortality &
\end{tabular}

The data were expressed as median (interquartile range) or case number (percentage). OHCA, out-of-hospital cardiac arrest.

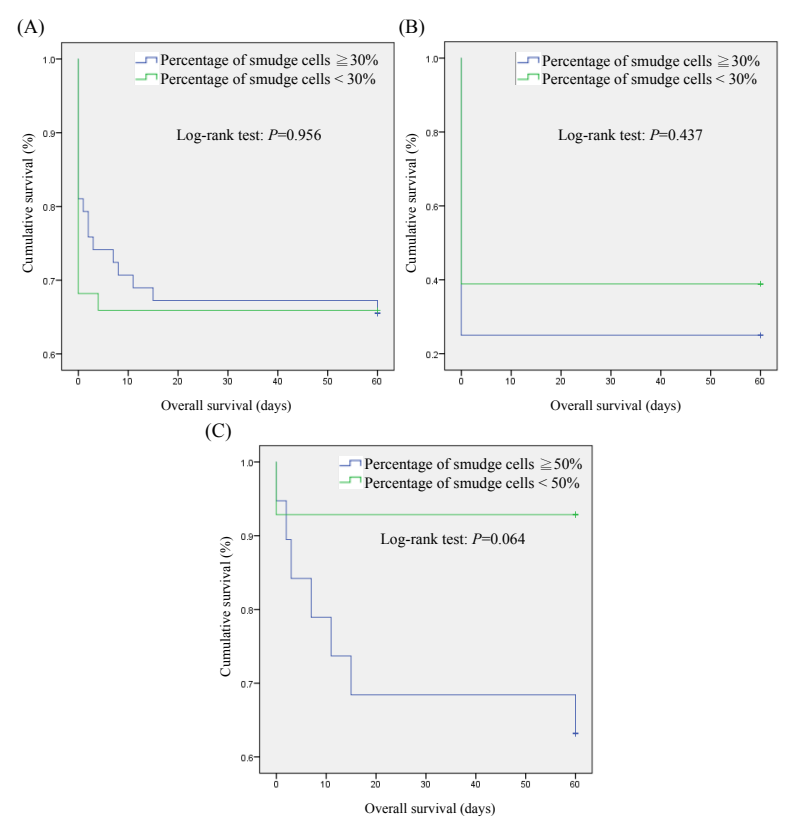

Figure 1. Survival analyses. Percentages of smudge cells and patient outcomes in those with smudge cells in the peripheral blood smear in all diseases (A), OHCA (B) and all cancers (C). OHCA, out-of-hospital cardiac arrest 
Table 2. Univariate Analysis for the Percentage of Smudge Cells And Patient Outcomes in those with Smudge Cells in the Peripheral Blood Film

\begin{tabular}{lcccc}
\hline Patient outcomes & The percentage of smudge cells & Crude OR (95\% CI) & P value \\
\hline All diseases & $\geq 30$ & $<30$ & $1.08(0.47-2.48)$ & 1.000 \\
Dead & 21 & 14 & & \\
Alive & 39 & 28 & $1.91(0.38-9.60)$ & 0.694 \\
OHCA & $\geq 30$ & $<30$ & & \\
Dead & 9 & 11 & & \\
Alive & 3 & 7 & $22.29(2.38-208.80)$ & \\
All cancers & $\geq 50$ & $<50$ & & N/A \\
Dead & 12 & 1 & & N/A \\
Alive & 7 & 13 & & N/A \\
Hematological malignancies & $\geq 50$ & $<50$ & & \\
Dead & 5 & 0 & & \\
Alive & 7 & 11 & & \\
Infections & $\geq 30$ & $<30$ & & \\
Dead & 0 & 2 & & \\
Alive & 12 & 9 & & \\
\hline OR & & & & \\
\end{tabular}

OR, odds ratio; $\mathrm{CI}$, confidence interval; N/A, not applicable

OR could not be calculated in patients with hematological cancers and infections because there was no death with the percentage of smudge cells less than $50 \%$ in hematological malignancies and with the percentage of smudge cells more than $30 \%$ in infections.

Figure 1 revealed the survival analysis of the percentage of smudge cells and patient outcomes in those with smudge cells in the peripheral blood smear in all diseases, OHCA and all cancers. There was no significant difference between the groups with smudge cells of $30 \%$ or more and less than $30 \%$ in all diseases $(\mathrm{P}=0.956$, Figure 1A) and OHCA $(\mathrm{P}=0.437$, Figure 1B), respectively. Additionally, it was seemingly that patients with smudge cells of $50 \%$ or more had a lower survival rate than those with smudge cells less than $50 \%$ in all cancers with follow-up in a 2-month interval, but with no statistical significance $(\mathrm{P}=0.064$, Figure $1 \mathrm{C})$.

\section{Discussion}

Our main finding indicated that smudge cells in the peripheral blood smear were frequently presented in OHCA, infections, hematological and solid cancers. It was shown that patients in all cancers with the percentage of smudge cell less than $50 \%$ had lower mortality rate when compared with those who had the percentage of smudge cells of $50 \%$ or more. Besides, it was seemingly that patients with smudge cells of $50 \%$ or more had a lower survival rate than those with smudge cells less than $50 \%$ in all cancers with follow-up in a 2-month interval. No apparent difference was found between the groups with smudge cells of $30 \%$ or more and less than $30 \%$ in all diseases and OHCA, respectively.

According to the literature review, smudge cells were prone to be observed in hematological malignancies, especially in chronic lymphocytic leukemia (Heinivaara et al., 1959; Nosanchuk et al., 1979; Paydas et al., 1999; Khandelwal et al., 2006; Carulli et al., 2007), although the presence of smudge cells had been considered as a well-described artifact before because of the rupture of fragile lymphocytes in preparing the peripheral blood smear (Macdonald et al., 2003). It was reported that a remarkable reduction in the percentage of smudge cells was found with the supplement of bovine serum albumin compared with those without (Lunning et al., 2004). Theoretically, the higher serum concentration of albumin should subsequently lead to less formation of smudge cells (Go et al., 2009). However, the association of percentage of smudge cells with serum albumin concentration of patients with chronic lymphocytic leukemia remained ascertained. Besides, accumulating evidence revealed that the percentage of smudge cells could be an independently prognostic indicator in patients with chronic lymphocytic leukemia (Nowakowski et al., 2009; Johansson et al., 2010; Gogia et al., 2014). In brief, it was shown that patients with more than $30 \%$ of smudge cells had a better overall survival rate than those with $30 \%$ or less smudge cells in chronic lymphocytic leukemia. Additionally, it was indicated that a high content of vimentin, one of the cytoskeletal proteins indispensable for the rigidity and integrity of lymphocytes, was significantly associated with lower percentage of smudge cells, as well as with a shortened interval to initial therapy in the early stage of chronic lymphocytic leukemia (Nowakowski et al., 2005). Further studies disclosed that there was an inverse correlation of the percentage of smudge cells with the presence of CD38, CD45 and zeta associated protein (ZAP)-70, which was also found to be independently prognostic factors for chronic lymphocytic leukemia (Nowakowski et al., 2007; Xu et al., 2009; Paydas, 2010; Zaher et al., 2011; Szerafin et al., 2012). The mutational status of immunoglobulin heavy chain and specific chromosomal aberrancies such as trisomy 12, 11q or $17 \mathrm{p}$ deletions were also considered as poor prognostic indicators for chronic lymphocytic leukemia (Wierda et al., 2011; Rizzo et al., 2013; Trajkova et al., 2013; Strati 
et al., 2014). In contrast, another study showed that there was no apparent correlation between the percentage of smudge cells in peripheral blood film and the prognosis of patients with chronic lymphocytic leukemia (Mozaheb et al., 2012). Interestingly, our investigation showed quite different results in which patients in all cancers with $50 \%$ or more smudge cells, including leukemias, had worse outcomes when compared with those with less than $50 \%$ of smudge cells in the peripheral blood smear. However, the underlying causes and mechanisms remained to be elucidated. One possible explanation to this discrepancy could be the divergence of race-ethnicity, since most of the study populations were non-Asian, in which patients diagnosed as chronic lymphocytic leukemia had better outcomes with higher percentage of smudge cells in the peripheral blood film. Therefore, a large-scale prospective study should be conducted to determine the prognostic role of smudge cells accompanied with the nutrition status in chronic lymphocytic leukemia, particularly in the Asian populations.

The presence of smudge cells in the blood film was also found in patients with OHCA and infections in our study. There was no obvious association of the percentage of smudge cells with the mortality or outcomes in perspective of all diseases, OHCA or all cancers. Whether the presence of smudge cells was related to the diseases rather than chronic lymphocytic leukemia remained ascertained.

The major limitation of our investigation was the retrospective study design with the limited subject number. Besides, the nutrition status was not available in the study population, making it difficult to differentiate the source of smudge cells formation from the categorized diseases per se or inappropriate preparation of blood film. Furthermore, the monitor interval could not be adequate enough to observe the disease evolution as well as the complication events and long-term mortality in certain disease categorizations, thus suggesting inevitable bias to some extents.

In summary, our study showed that in all cancers, those who had higher percentage of smudge cells were prone to have poor outcomes when compared with the subjects with lower percentage of smudge cells in the blood smear. This finding was quite different from the results of previous studies in which the race-ethnicity of most study populations was non-Asian. Hence, the clinical significance of smudge cells in malignancies remained indeterminate and further investigations were required. Besides, there was no apparent association of the percentage of smudge cells with patient outcomes in all diseases and OHCA, respectively.

\section{References}

Carulli G, Marini A, Baccelli E, et al (2007). Association of B-chronic lymphocytic leukemia and acute myeloid leukemia. J Exp Clin Cancer Res, 26, 421-4.

Go RS (2009). Smudge cells, serum albumin, and prognosis in B-cell chronic lymphocytic leukemia. J Clin Oncol, 27, 44.

Gogia A, Raina V, Gupta R, et al (2014). Prognostic and predictive significance of smudge cell percentage on routine blood smear in chronic lymphocytic leukemia. Clin
Lymphoma Myeloma Leuk, 14, 514-7

Gumprecht F (1986). Leucocytenzerfall in blute bal leukemic und bel sohwaren anamlan. Deutches Archlv Kinlache Medicin, 5, 523-48.

Heinivaara O (1959). Smudge cells in lymphocytic leukemia. Ann Med Intern Fenn, 48, 69-75.

Johansson P, Eisele L, Klein-Hitpass L, et al (2010). Percentage of smudge cells determined on routine blood smears is a novel prognostic factor in chronic lymphocytic leukemia. Leuk Res, 34, 892-8.

Khandelwal A, Seilstad KH, Magro CM (2006). Subclinical chronic lymphocytic leukaemia associated with a $13 q$ deletion presenting initially in the skin: apropos of a case. $J$ Cutan Pathol, 33, 256-9.

Lunning MA, Zenger VE, Dreyfuss R, et al (2004). Albumin enhanced morphometric image analysis in CLL. Cytometry B Clin Cytom, 57, 7-14.

Macdonald D, Richardson H, Raby A (2003). Practice guidelines on the reporting of smudge cells in the white blood cell differential count. Arch Pathol Lab Med, 127, 105.

Mozaheb Z, Hasanzadeh NazarAbadi MH, Aghaee MA (2012). Chronic lymphocytic leukemia and prognostic factors. Asian Pac J Cancer Prev, 13, 3009-13.

Nosanchuk JS (1979). The effect of smudge cells on leukocyte counting--are chamber counts necessary? Am J Clin Pathol, 71, 161-6.

Nowakowski GS, Lee YK, Bone ND, et al (2005). Proteomic analysis of chronic lymphocytic leukemia cells identifies vimentin as a novel prognostic factor for aggressive disease. Blood, 106, 707.

Nowakowski GS, Hoyer JD, Shanafelt TD, et al (2007). Using smudge cells on routine blood smears to predict clinical outcome in chronic lymphocytic leukemia: A universally available prognostic test. Mayo Clin Proc, 82, 449-53.

Nowakowski GS, Hoyer JD, Shanafelt TD, et al (2009). Percentage of smudge cells on routine blood smear predicts survival in chronic lymphocytic leukemia. J Clin Oncol, 27, 1844-9.

Paydas S (1999). Interesting morphologic finding in T-cell acute lymphoblastic leukemia. Diagn Cytopathol, 21, 55-6.

Paydas S (2010). Smudge cells: very old history and new conclusions. Leuk Res, 34, 1680.

Rizzo D, Lotay A, Gachard N, et al (2013). Very low levels of surface CD45 reflect CLL cell fragility, are inversely correlated with trisomy 12 and are associated with increased treatment-free survival. Am J Hematol, 88, 747-53.

Strati P, Keating MJ, O'Brien SM, et al (2014). Outcomes of first-line treatment for chronic lymphocytic leukemia with 17p deletion. Haematologica, 99, 1350-5.

Szerafin L, Jakó J, Riskó F, et al (2012). The prognostic value of smudge cells (Gumprecht shadows) in chronic lymphocytic leukaemia. Orv Hetil, 153, 1732-7.

Trajkova S, Cevreska L, Pivkova-Veljanovska A, et al (2013). Multivariable model consisting of clinical and biological markers for time to first treatment in CLL patients: Preliminary results from single centre experience. Prilozi, 34, 39-48.

Wierda WG, O'Brien S, Wang X, et al (2011). Multivariable model for time to first treatment in patients with chronic lymphocytic leukemia. J Clin Oncol, 29, 4088-95.

$\mathrm{Xu} \mathrm{W,} \mathrm{Li} \mathrm{J,} \mathrm{Wu} \mathrm{Y,} \mathrm{et} \mathrm{al} \mathrm{(2009).} \mathrm{CD38} \mathrm{as} \mathrm{a} \mathrm{prognostic} \mathrm{factor} \mathrm{in}$ Chinese patients with chronic lymphocytic leukemia. Leuk Res, 33, 237-43.

Zaher A, Elgamal B (2011). Peripheral blood smudge cells percentage in de novo CLL: a comparison with other stablished laboratory prognostic markers. Life Sci J, 8, 239-44. 